\title{
Applications of Atomic-Resolution EELS Mapping at Low kV
}

\author{
B. Schaffer, $* * * \quad$ F. Azough, ${ }^{* * *} \quad$ D. Abou-Ras, ${ }^{* * * *}$, S.S. Schmidt, $* * * * \quad$ M. Schaffer, ${ }^{* * * * * *}$ \\ M. Sarahan, ${ }^{* * *}$, Q. Ramasse, ${ }^{* * * * * *}$ and R. Caballero*** \\ * SuperSTEM, STFC Daresbury Laboratories, Keckwick Lane, Warrington, WA4 4AD, UK \\ ** Kelvin Nanocharacterisation Centre, SUPA School of Physics and Astronomy, University of \\ Glasgow, Glasgow, G12 8QQ, Scotland, UK \\ *** Materials Science Centre, School of Materials, University of Manchester, Manchester M1 7HS, \\ UK \\ **** Helmholtz-Zentrum Berlin, Hahn-Meitner-Platz 1, 14109 Berlin, Germany \\ ***** Department of Engineering, George Holt Building, Ashton Street, Liverpool, L69 3BX, UK
}

In recent years, aberration corrected scanning transmission electron microscopy (STEM) has become a widely used technique in materials science, which routinely provides atomic-resolution Z-contrast imaging. The possibility to combine image detectors with analytical detectors for simultaneous mapping of these signals at similar sub-atomic spatial sampling gives STEM a distinct advantage over other atomic-resolution imaging techniques. 2D chemical maps of individual atomic columns have been demonstrated using electron energy-loss spectroscopy (EELS) [1-5] and, more recently, energy-dispersive X-ray spectroscopy (EDXS) [6].

As high probe currents and large EELS detection collection angles have become available in new instruments, the limiting parameter in applications is often the sample itself. Outstanding sample preparation is paramount. Even with perfectly prepared samples, beam damage and specimen drift induced by electron beam interactions often dictate a narrow set of suitable experimental parameters for 2D mapping, limiting the attainable signal-to-noise (SNR) ratio. Clearer maps can often be obtained by applying post-processing techniques such as principal component analysis (PCA), multiple linear least-square (MLLS) fitting of references, or by other multivariate statistical analysis techniques [7-9]. Such techniques are explored and developed in house.

Fig. 1 shows an example of 2D EELS SI data of a $\mathrm{Ba}_{6-3 x} \mathrm{Nd}_{8+2 \mathrm{x}} \mathrm{Ti}_{18} \mathrm{O}_{54}$ ceramic. The 2D EELS SI data set was acquired with the aberration corrected NION UltraSTEM at SuperSTEM/Daresbury equipped with a Gatan ENFINA electron spectrometer and operated at $100 \mathrm{kV}$.

Recently, the material class of $\mathrm{sp}^{2}$-bonded monoatomic layers has gained strong attention. Many of these samples can not withstand electron irradiation sufficiently long to facilitate reliable EELS mapping at higher acceleration voltages. Reducing the voltage to $60 \mathrm{kV}$ or below has proven a successful strategy to produce atomically resolved 2D EELS maps with the UltraSTEM in our lab. However, if beam damage prevents even 1D line-scan acquisitions of sufficient SNR, distributeddose acquisitions may also be applicable. The Smart Acquisition [10] routine scans the beam rapidly through a set of pre-defined positions during acquisition of a single spectrum. While some spatial information is sacrificed in the averaged spectrum, the increased exposure time or reduced beam damage often reveals otherwise inaccessible information.

A typical application of the Smart Acquisition approach is the averaged line-scan as shown in Fig.2. Here, elemental profiles of a $\mathrm{Cu}(\mathrm{In}, \mathrm{Ga}) \mathrm{Se}_{2}$ thin film across grain boundaries and defects can be gained with sufficient SNR. Notably, the atomic resolution is retained in the profile direction.

In this work, we present the application of atomic resolution EELS analysis on different materials systems, highlighting obstacles and solutions of the technique. 


\section{References}

[1] M. Bosman et al., PRL 99 (2007) 086102.

[2] K. Kimoto et al., Nature 450 (2007) 702.

[3] D.A. Muller et al., Science 319 (2008) 1073.

[4] Varela et al., PhysRev B 79 (2009) 085117.

[5] G.A. Botton et al., Ultramicroscopy (2010) 926.

[6] M. Watanabe et al., Jeol News 45/1 (2010) 8.

[7] N. Bonnet et al., Ultramicroscopy (1999) 97.

[8] M. Sarahan et al., Ultramicroscopy (2011) 251.

[9] F. de la Pena et al., Ultramicroscopy (2011) 169.

[10] K. Sader et al., Ultramicroscopy (2010) 998.

[11] The authors would like thank EPSRC for funding under grant EP/D040205/1.

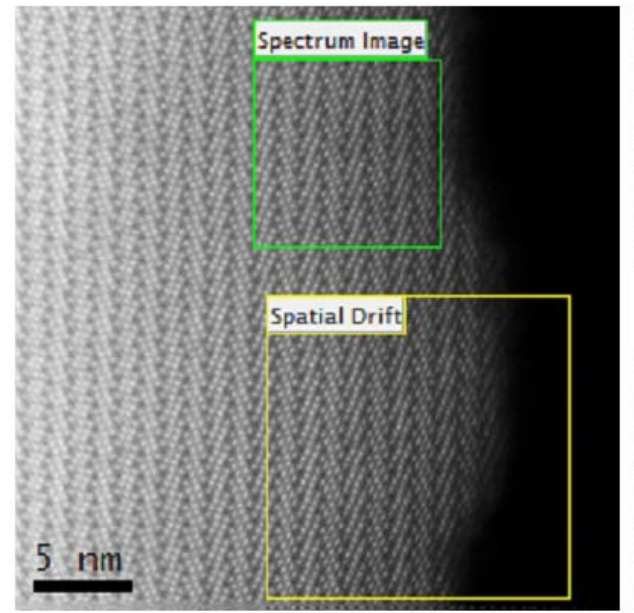

HAADF survey

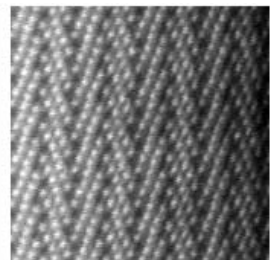

HAADF survey

(SI detail)

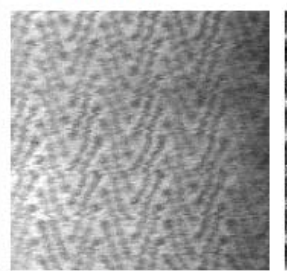

Ti map

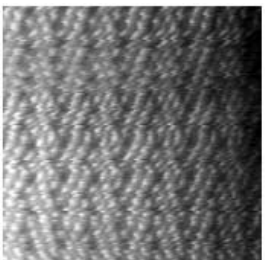

HAADF

simultaneous

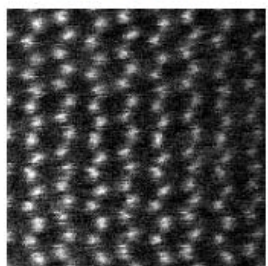

Ba map

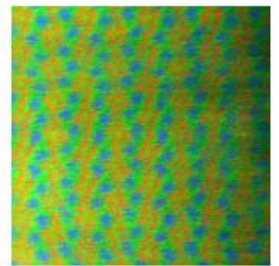

composition map $(\mathrm{R} / \mathrm{G} / \mathrm{B}=\mathrm{Nd} / \mathrm{Ti} / \mathrm{Ba})$

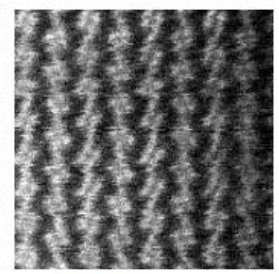

Nd map

FIG. 1. Example of atomically resolved 2D EELS mapping of a $\mathrm{Ba}_{6-3 x} \mathrm{Nd}_{8+2 \mathrm{x}} \mathrm{Ti}_{18} \mathrm{O}_{54}$ ceramic.

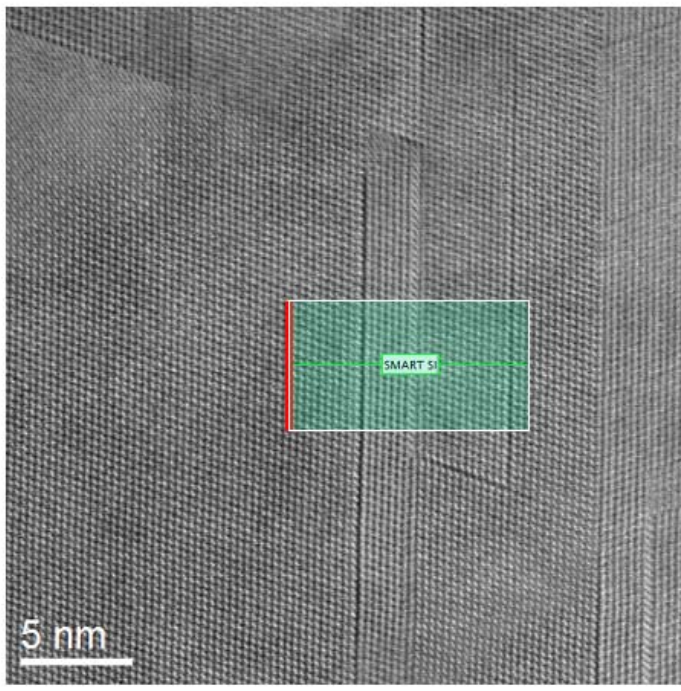

HAADF with schematic SMART SI line-profile marker. The line profile is acquired from left to right in 345 steps. At each step the beam is rapidly scanned up and down as indicated by the red bar.

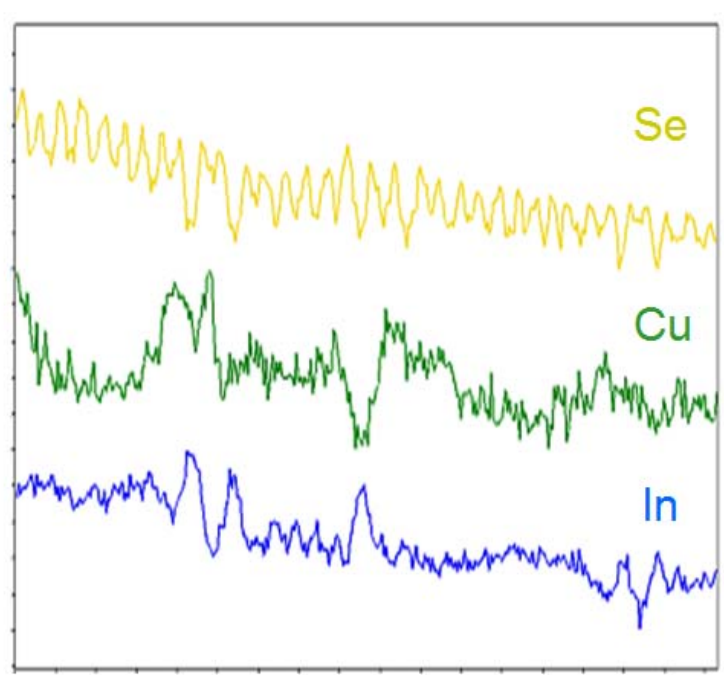

Line profiles of In, $\mathrm{Cu}$, Se derived from the SMART SI data

FIG. 2. Example of a Smart Acquisition line scan of a $\mathrm{Cu}(\mathrm{In}, \mathrm{Ga}) \mathrm{Se}_{2}$ thin film in a solar cell. Atomic resolution in scan profile is given, while the profile is averaged in perpendicular direction. 